\title{
Erratum to: Extrinsic diameter of immersed flat tori in $S^{3}$
}

\author{
Yoshihisa Kitagawa · Masaaki Umehara
}

Received: 23 May 2013 / Accepted: 7 August 2013 / Published online: 1 September 2013

C) Springer Science+Business Media Dordrecht 2013

\begin{abstract}
In the paper referred to in the title, we proved that any immersed flat tori in $S^{3}$ whose mean curvature does not change sign has extrinsic diameter $\pi$. Although the main result there is correct, there is a gap of the proof of this fact. The purpose here is to correct the previous paper's argument and clarify the statement.
\end{abstract}

Mathematics Subject Classification (2000) Primary 53C42 - Secondary 53C40 · 53C75

\section{Introduction}

Let $S^{3}$ be the unit sphere in $\mathbf{R}^{4}=\mathbf{C}^{2}$. The Clifford torus in $S^{3}$ given by

$$
M_{\theta}:=\left\{(z, w) \in C^{2} ;|z|^{2}=\cos ^{2} \theta, \quad|w|^{2}=\sin ^{2} \theta\right\}, \quad 0<\theta<\pi / 2,
$$

is a flat embedded torus of constant mean curvature. We denote by $\iota_{\theta}: M_{\theta} \rightarrow S^{3}$ the canonical inclusion, and by $d s_{\theta}^{2}$ is the induced metric on $M_{\theta}$. In the previous paper [1, Theorem 0.2], we stated the following assertion:

Theorem Let $f:\left(M_{\theta}, d s_{\theta}^{2}\right) \rightarrow S^{3}$ be an isometric immersion whose mean curvature function does not change sign on $M_{\theta}$, then $f$ is congruent to $\iota_{\theta}$.

The online version of the original article can be found under doi:10.1007/s10711-011-9580-5.

\footnotetext{
Y. Kitagawa

Department of Mathematics, Utsunomiya University, Mine-machi, Utsunomiya 321-8505, Japan

e-mail: kitagawa@cc.utsunomiya-u.ac.jp

M. Umehara $(\varangle)$

Department of Mathematical and Computing Sciences, Tokyo Institute of Technology, 2-12-1-W8-34, O-okayama, Meguro-ku, Tokyo 152-8552, Japan

e-mail: umehara@is.titech.ac.jp
} 
Fig. 1 A typical case where the previous argument fails

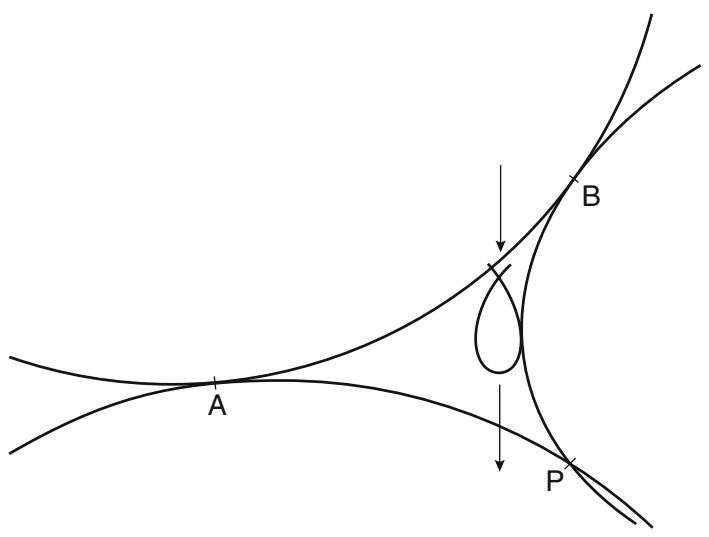

Unfortunately, there was a gap in the proof of Proposition 4.7, which was a key assertion to prove the theorem. In fact, in [1, Claim 5 (Page 133)], we stated that the subset $\mathcal{L}$ is closed in the subarc $\left.C\right|_{[A, B]}$ of $C$. However, this is not true if $s_{\infty}$ does not coincide with $s_{X_{\infty}}$. In fact, $s=s_{\infty}$ might not be the first point where $\varphi_{s}\left(S_{2}^{\prime}\right)$ meets the union of the $\operatorname{arcs}[A P) \cup[B P)$, where $[A P)$ and $\left[B P\right.$ ) are subarcs of $\gamma_{1}$ defined in [1, Page 132]. For example, if we consider the case as in Fig. 1 , the set $\mathcal{L}$ is not closed, since the shell is tangent to $(P B]$ before attaching to $[A P)$. The purpose of this paper is to correct the proof of Proposition 4.7.

\section{A key proposition}

The following assertion will play a crucial role in the new proof of Proposition 4.7. (The definition of positive shell and negative shell is given in [1, Page 118].)

Proposition 1.1 Let $\sigma:[0, a] \rightarrow S^{2}$ be a positive shell (respectively negative shell) whose geodesic curvature $\kappa(t)(0 \leq t \leq a)$ satisfies $\kappa(t)>\kappa_{0}$ (respectively $\left.\kappa(t)<-\kappa_{0}\right)$, where $\kappa_{0}$ is a positive constant. Let $\Gamma$ be the circle of geodesic curvature $\kappa_{0}$ (respectively $\left.-\kappa_{0}\right)$ which is tangent to $\sigma(t)$ at $t=b(0 \leq b \leq a)$. Then $\sigma([0, a]) \backslash\{\sigma(b)\}$ lies in the interior $\Delta_{\Gamma}$ of the circle $\Gamma$ (the definition of $\Delta_{\Gamma}$ is given in [1, Page 118]).

To prove this, we show the following lemma, which is a special case of the proposition.

Lemma 1.2 Let $\sigma:[0, a] \rightarrow S^{2}$ be a positive shell whose geodesic curvature $\kappa(t)(0 \leq$ $t \leq a)$ satisfies $\kappa(t)>\kappa_{0}$, where $\kappa_{0}$ is a positive constant. Let $\Gamma$ be the circle of geodesic curvature $\kappa_{0}$ which is tangent to $\sigma(t)$ at $t=0$ or $t=a$. Then $\sigma((0, a))$ lies in the interior $\Delta_{\Gamma}$ of the circle $\Gamma$.

Proof We consider the case $t=0$. (The case $t=a$ is proved similarly.) Suppose that the image $\sigma((0, a))$ does not lie in $\Delta_{\Gamma}$. The condition $\kappa(t)>\kappa_{0}$ yields that $\sigma(t)$ lies in $\Delta_{\Gamma}$ for sufficiently small $t>0$. Since $\sigma$ is a positive shell, $\sigma(a-t)$ also lies in $\Delta_{\Gamma}$ for sufficiently small $t>0$. Thus, there exist two points $s_{1}, s_{2} \in(0, a)$ satisfying the following three properties (see Fig. 2, left):

- $0<s_{1} \leq s_{2}<a$,

- $\sigma\left(s_{1}\right)$ and $\sigma\left(s_{2}\right)$ lie on $\Gamma$,

- $\sigma\left(\left(0, s_{1}\right)\right)$ and $\sigma\left(\left(s_{2}, a\right)\right)$ are contained in the domain $\Delta_{\Gamma}$. 

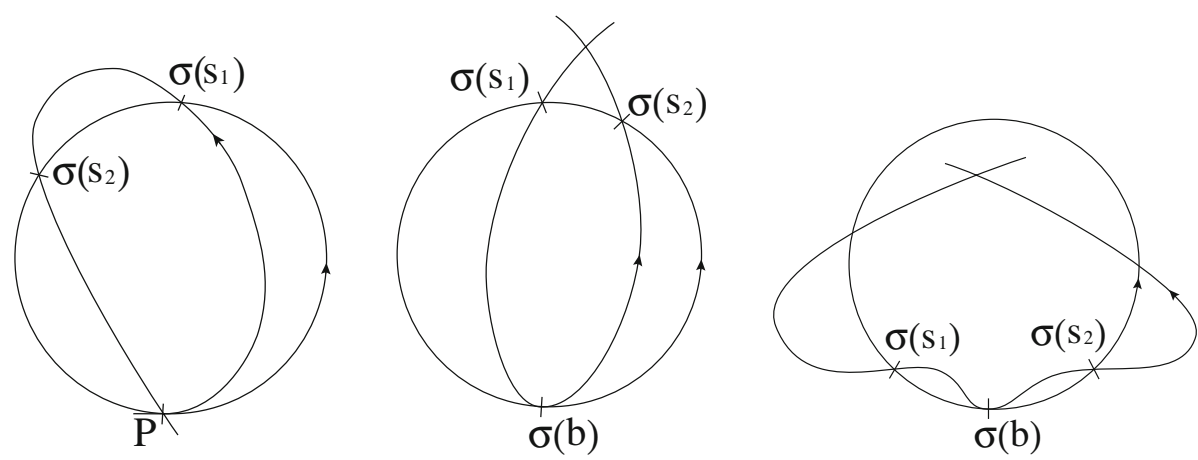

Fig. 2 Three impossible arrangements of $\sigma$
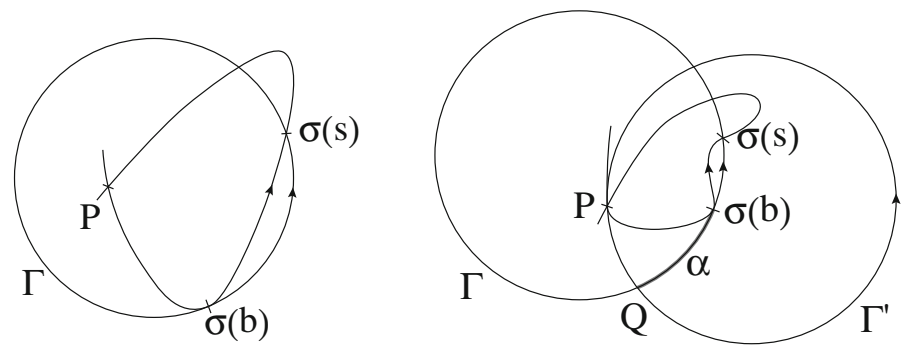

Fig. 3 The case $\sigma((0, b)) \subset \Delta_{\Gamma}$

Applying [1, Lemma 4.5] to the arc $\sigma\left(\left[0, s_{1}\right]\right)$, we can conclude that $\sigma\left(s_{1}\right)$ lies in the past part of $\Gamma$ with respect to the node $P:=\sigma(0)=\sigma(a)$. Since $\sigma$ is a simple closed arc, $\sigma\left(s_{2}\right)$ must also lie in the past part of $\Gamma$ with respect to $P$. On the other hand, applying [1, Lemma 4.5] to another $\operatorname{arc} \sigma\left(\left[s_{2}, a\right]\right)$, we can conclude that $\sigma\left(s_{2}\right)$ must lie in the future part of $\Gamma$ with respect to $P$, which is a contradiction.

We now prove Proposition 1.1 as follows: Reversing the orientation of the curve $\sigma$ if necessary, we may assume that $\sigma$ is a positive shell. Since Lemma 1.2 proves the case of $b=0$ or $b=a$, we may assume that $0<b<a$. Suppose also that the image $\sigma([0, a]) \backslash\{\sigma(b)\}$ does not lie in $\Delta_{\Gamma}$. The center and right of Fig. 2 are typical such cases. Suppose that both $\sigma([0, b))$ and $\sigma((b, a])$ do not lie in $\Delta_{\Gamma}$. Then, there exist two points $s_{1}, s_{2} \in[0, a]$ satisfying the following three properties:

- $0 \leq s_{1}<b<s_{2} \leq a$,

- $\sigma\left(s_{1}\right)$ and $\sigma\left(s_{2}\right)$ lie on $\Gamma$,

- $\sigma\left(\left(s_{1}, b\right)\right)$ and $\sigma\left(\left(b, s_{2}\right)\right)$ are contained in the domain $\Delta_{\Gamma}$.

We can make a contradiction applying [1, Lemma 4.5] for two $\operatorname{arcs} \sigma\left(\left[s_{1}, b\right]\right)$ and $\sigma\left(\left[b, s_{2}\right]\right)$, respectively, like as in the proof of Lemma 1.2. Thus, either $\sigma([0, b))$ or $\sigma((b, a])$ lies in $\Delta_{\Gamma}$. In particular, $P$ lies in $\Delta_{\Gamma}$. We now consider the case that $\sigma([0, b))$ lies in $\Delta_{\Gamma}$. (The case $\sigma((b, a]) \subset \Delta_{\Gamma}$ also makes a contradiction using the same argument.) Then, there exists a point $s \in(b, a)$ such that $\sigma(s)$ lies on $\Gamma$ and $\sigma((b, s))$ lies in $\Delta_{\Gamma}$ (see Fig. 3, left). Let $\Gamma^{\prime}$ be the circle of geodesic curvature $\kappa_{0}$ which is tangent to $\sigma(t)$ at $t=0 . \Gamma^{\prime}$ intersects $\Gamma$ with two points. Let $Q$ be one of two such intersection points, which lies in the past part of $\Gamma$ with respect to $\sigma(b)$. By Lemma 1.2, $\sigma((0, a))$ lies in $\Delta_{\Gamma^{\prime}}$ (cf. Fig. 3, right). In particular, $\sigma(b)$ 
and $\sigma(s)$ lie on $\Gamma \cap \Delta_{\Gamma^{\prime}}$. Then, we can conclude that $\sigma(b)$ and $\sigma(s)$ lie in the future part of $\Gamma$ with respect to $Q$. On the other hand, applying [1, Lemma 4.5] to the arc $\sigma([b, s])$, we can conclude that $\sigma(s)$ lies in the past part of $\Gamma$ with respect to $\sigma(b)$. Hence $\sigma(s)$ must lie in the subarc $\alpha$ of $\Gamma$ bounded by $Q$ and $\sigma(b)$ (see Fig. 3, right). However, this implies that $\sigma((b, s))$ must meet the arc $\sigma([0, b])$, which contradicts the fact that $\sigma$ is a simple closed arc. This proves the proposition.

\section{A modification of the definition of $r$-diamonds}

In [1, Definition 4.8], we gave a definition of $r$-diamonds. However, we need to modify it for the new proof of Proposition 4.7. More precisely, the condition (v) of $r$-diamonds should be replaced by the following condition:

$\left(\mathrm{v}^{\prime}\right)$ Let $C$ be the circle of radius $r$ centered at $O$, which is tangent to $\gamma_{1}$ at $A$ and $B$. We denote by $[A P]$ (respectively $[B P]$ ) the subarc of $\gamma_{1}$ bounded by $A$ (respectively $B)$ and $P$. We also use another notion $[A P$ ) (respectively $[B P)$ ) which contains the end point $A$ (respectively $B$ ) but does not contain the other end point $P$. Fix $X \in[A P$ ) and $Y \in\left[B P\right.$ ). Then there exists a circle $C_{X}$ (respectively $C_{Y}$ ) of radius $\delta_{\mu}$ which is tangent to $[A P)$ at $X$ (respectively $[B P)$ at $Y$ ) and $(X P]$ (respectively $(Y P])$ lies in $\Delta_{C_{X}}$ (respectively $\Delta_{C_{Y}}$ ), where $\Delta_{C_{X}}$ and $\Delta_{C_{Y}}$ are the interior domains of the circles $C_{X}$ and $C_{Y}$, respectively.

From now on, we replace condition (v) with this new modification for the definition of $r$-diamonds. (All the other conditions (i)-(iv) remain as before. The previous condition (v) is the special case of the new condition $\left(\mathrm{v}^{\prime}\right)$ by putting $X=A$ and $Y=B$.) Thus, our $r$-diamonds satisfy the old definition of $r$-diamonds in [1], but the converse is not true. By this change, two points $A, B$ can move not only on $S_{1}$ but also whole on $\gamma_{1}$ (see [1, (4.9)] for the definition of $S_{1}$ ).

In Claim 1-3 in [1, Section 4], we proved that there is a $\delta_{\mu}^{*}$-diamond $\diamond_{O A P B}$. This proof can apply for our new definition of $r$-diamonds by a suitable modification as follows: Since the curvature radius of each point of the spherical curve $\gamma_{1}$ is less than $\delta_{\mu}$ (cf. [1, (4.7)]), the condition $\left(\mathrm{v}^{\prime}\right)$ holds for the 4-gon $O A P B$ as in [1, Fig. 17] for sufficiently small $r>0$. By replacing $S_{1}$ to $\gamma_{1}$, the proofs of Claim 1-2 are valid even if $A, B \notin S_{1}$. We now pay attention to the sequence of $r_{n}$-diamonds $\left\{\diamond_{O_{n} A_{n} P B_{n}}\right\}_{n=1,2, \ldots}$ in the proof of Claim 3 . For the sake of simplicity, we set $A:=A_{\infty}$ and $B:=B_{\infty}$. From now on, we show that $[A P]$ and $[B P]$ are both simple arcs: In fact, there exist $c_{1}, c_{2} \in S^{1}:=\boldsymbol{R} / \boldsymbol{Z}$ such that $\gamma_{1}\left(c_{1}\right)=P$ and $\gamma_{1}\left(c_{2}\right)=A$. Since $\diamond_{O_{n} A_{n} P B_{n}}$ is a diamond, $\left[A_{n} P\right]$ is a simple arc. So if we suppose that $[A P]$ has a self-intersection, then there exists $m \in\left(c_{1}, c_{2}\right)$ such that $A=\gamma_{1}(m)$. Since all of the crossings on $\gamma_{1}$ are transversal, either $\gamma_{1}(m-\varepsilon)$ or $\gamma_{1}(m+\varepsilon)$ does not lie in the interior domain $\Delta_{C_{A_{n}}}$ for a sufficiently small $\varepsilon>0$ and for sufficiently large $n$. However, it contradicts the condition $\left(\mathrm{v}^{\prime}\right)$ of the diamond $\diamond_{O_{n} A_{n} P B_{n}}$, since $\gamma_{1}(m)$ lies in $\left[A_{n} P\right.$ ) for sufficiently large $n$. Similarly, $[B P]$ is also a simple arc.

We now suppose that the limit 4-gon $O_{\infty} A P B$ is not a $\rho$-diamond $\left(\rho:=\delta_{\mu}^{*}\right)$.

Then, by the condition $\left(\mathrm{v}^{\prime}\right)$, there exists $X \in[A P)$ or $Y \in[B P)$ such that either $(X P]$ meets $C_{X}$ or $(Y P]$ meets $C_{Y}$. Without loss of generality, we may consider the case $(X P]$ meets $C_{X}$. If $X \neq A$, then $X \in\left(A_{n} P\right]$ holds for a sufficiently large $n$. Then, the property $\left(\mathrm{v}^{\prime}\right)$ of the $r_{n}$-diamond $\diamond_{O A_{n} P B_{n}}$ yields that $C_{X}$ does not meet $(X P$ ], a contradiction.

So we may assume that $X=A$. Now it is sufficient to make a contradiction under the assumption that $(A P]$ meets $C_{A}$. Since $\left(A_{n} P\right]$ lies in the domain $\Delta_{C_{A_{n}}}$, by taking limit 
Fig. 4 A figure which should added in [1, Figure 9]

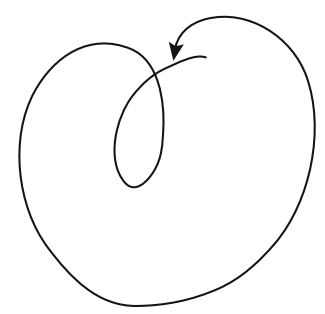

$n \rightarrow \infty$, there exists a point $Q$ on $(A P]$ such that $Q$ lies on the circle $C_{A}$. Without loss of generality, we may assume that $Q$ is the first such point, namely, $(A Q)$ does not meet $C_{A}$. (see [1, Fig. 20, left and right]). We give an orientation of $C_{A}$ so that it has positive geodesic curvature. Since $C_{A}$ is of radius $\delta_{\mu}$, the geodesic curvature of $\gamma_{1}$ is greater than that of $C_{A}$. Then [1, Lemma 4.5] implies that $Q$ lies in the future part of $C_{A}$ with respect to $A$.

We first consider the case $Q=P$. Consider the subarc of $C_{A}$ defined by

$$
\mathfrak{a}:=C_{A} \cap \overline{\Delta_{C_{B}}} .
$$

Then $\mathfrak{a}$ lies in the past part of $C_{A}$ with respect to $A$. Since $Q$ lies in the subarc a, it contradicts the fact that $Q$ lies in the future part of $C_{A}$.

We next consider the case $Q \neq P$. We denote by $C_{A}^{+}$(respectively $C_{A}^{-}$) the future part (respectively the past part) of $C_{A}$ with respect to $A$. Since $Q \in C_{A}^{+}$, we can consider the subarc $\mathfrak{b}$ of $C_{A}^{+}$bounded by $Q$ and $A$. Since $\mathfrak{a} \subset C_{A}^{-}$, the two circular arcs $\mathfrak{a}$ and $\mathfrak{b}$ are disjoint. Since the geodesic curvature of $\gamma_{1}(t)$ at $Q$ is greater than $k_{0}$, the subarc [ $\left.Q P\right]$ of $\gamma_{1}$ lies on the domain $D$ bounded by $\mathfrak{b}$ and $[A Q$ ) (see [1, Fig. 20], right). Since $P$ lies in $\bar{D}$ and $[B P$ ) never meets $[A P)$, there is a point $R$ on $[B P) \cap \mathfrak{b}$. On the other hand, since $[B P] \subset \overline{\Delta_{C_{B}}}$, we have that

$$
R \in \mathfrak{a},
$$

which contradicts that $\mathfrak{a}$ and $\mathfrak{b}$ are disjoint.

Remark 2.1 In [1, Figure 9], we showed the two possibilities of the behavior of the curve $\gamma_{1}$. However, the case as in Fig. 4 was missing in [1, Figure 9] as the third possibility. Fortunately, this is just the case of $\Theta_{C}$ as in [1, Figure 11], the statement and the proof of [1, Corollary 3.11] themselves do not need any corrections.

\section{The proof of Proposition 4.7}

For the correction of the proof of Proposition 4.7, we also need the following renewal of the arguments given in [1, Page 131 Line 15-Page 133 Line 21]: We set $\rho:=\delta_{\mu}^{*}$. Let $\diamond_{O A P B}$ be the $\rho$-diamond as in Section 2, and $C$ the circle of radius $\rho$ centered at $O$. By [1, Corollary 3.7], there exists a negative shell $S_{2}$ on $\gamma_{2}$. We place $S_{2}$ so that it lies inside of the circle C of radius $\rho$, and the node of $S_{2}$ is tangent to the $\operatorname{arc}[B P$ ) at $B$ (see Fig. 5, left). By Proposition $1.1, S_{2}$ actually lies inside of the circle $C$. We slide the shell $S_{2}$ along the arc $[B P)$. We denote by $S_{2}(Y)$ this sliding shell whose node lies at $Y$ for each $Y \in[B P)$. When $Y=B$, $S_{2}(Y)$ coincides with $S_{2}$.

Consider the baroon-like closed domain $\bar{\Omega}$ bounded by three $\operatorname{arcs}[A P],[B P]$ and $A B$, where $A B$ is the major arc on $C$ bounded by $A, B$. By Proposition 1.1, the shell $S_{2}$ lies in $\bar{\Omega}$ (cf. Fig. 5, left). Let $Z$ be the first point at which $S_{2}(Y) \backslash\{Y\}$ meets the boundary of $\bar{\Omega}$. 

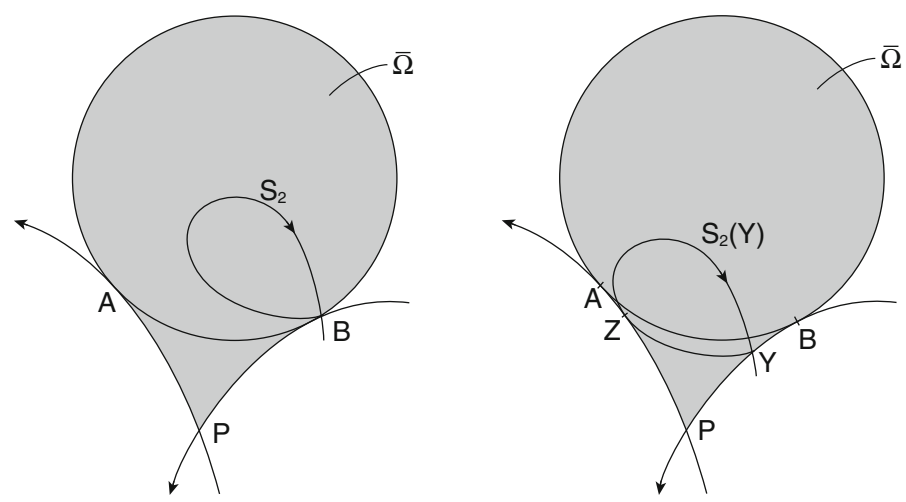

Fig. 5 The sliding operation of $S_{2}$

If $Z$ lies on the circle $C$, then whole of $S_{2}(Y) \backslash\{Z\}$ lies in $\Delta_{C}$ by Proposition 1.1, which contradicts the fact that $Z \neq Y$ and $Y \in[B P$ ). So $Z$ lies in [AP) or [ $B P$ ). If $Z$ lies in $[A P$ ), then $(Y, Z)$ gives an admissible bi-tangent (cf. [1, Definition 2.1.] and Fig. 5, right), which proves Proposition 4.7. So it is sufficient to make a contradiction if $Z$ lies in $[P B)$.

We first consider the case $Z \in(Y P]$. By Proposition 1.1, there exists a circle $\Gamma$ of radius $\rho$ which is tangent to $[B P)$ at $Y$ such that $S_{2}(Y) \backslash\{Y\}$ lies in $\Delta_{\Gamma}$. In particular, $Z \in \Delta_{\Gamma}$. Since $(Y P]$ lies in the interior $\Delta_{C_{Y}}$ of the circle $C_{Y}$ by the condition $\left(\mathrm{v}^{\prime}\right)$ of diamonds, we can conclude that $\Delta_{C_{Y}} \cap \Delta_{\Gamma}$ is an empty set. Thus $Z \notin \Delta_{C_{Y}}$. However, this contradicts the fact that $(Y P]$ lies in $\Delta_{C_{Y}}$.

We next consider the other case $Z \in[B Y)$. By Proposition 1.1, there exists a circle $\Gamma^{\prime}$ of radius $\rho$ which is tangent to $[B P)$ at $Z$ such that $S_{2}(Y) \backslash\{Z\}$ lies in the domain $\Delta_{\Gamma^{\prime}}$. In particular, $Y \in \Delta_{\Gamma^{\prime}}$. Since $(Z P]$ is contained in $\Delta_{C_{Z}}$ by the condition $\left(\mathrm{v}^{\prime}\right)$ of diamonds, $\Delta_{C_{Z}} \cap \Delta_{\Gamma^{\prime}}$ is an empty set. Thus $Y \notin \Delta_{C_{Z}}$, which contradicts the fact that $(Z P]$ lies in $\Delta_{C_{Z}}$.

All of the other arguments in [1] are correct without any modifications.

\section{Reference}

1. Kitagawa, Y., Umehara, M.: Extrinsic diameter of immersed flat tori in $S^{3}$. Geom. Dedicata 155, 105-140 (2011) 